\title{
Les premières étudiantes à la Faculté de médecine et leurs activités professionnelles à Genève
}

Par Jean Jacques Dreifuss

RÉsumé

Dès ses débuts la Faculté de médecine de l'Université de Genève accueillit de nombreuses étudiantes, notamment d'origine slave. Une fois leurs études terminées, peu de ces femmes médecins demeuraient à Genève pour y pratiquer, car l'environnement professionnel leur était plutôt hostile. Trois carrières sont décrites qui illustrent les difficultés rencontrées par celles qui demeurèrent à Genève et les activités qu'elle exercèrent. Henriette de Joudra (1856-1928) épousa un confrère suisse et mena de pair une vie de famille et la pratique de la médecine; la Société médicale de Genève refusa cependant de l'admettre comme membre. Marguerite Champendal (1870-1928), une des premières étudiantes suisses, se consacra à l'hygiène de la mère et de l'enfant et fonda l'école d'infirmières Le Bon Secours. Biochimiste et physiologiste, Lina Stern (1878-1968) fut en 1918 la première femme à professer à la Faculté de médecine de Genève, avant de poursuivre sa carrière académique dès 1925 en Union soviétique.

On sait que Zurich fut la première université suisse à ouvrir les portes de sa Faculté de médecine aux femmes et que deux jeunes Russes y étudièrent la médecine dès 1865. Trois ans plus tard, elles étaient déjà au nombre de huit, dont une première Suissesse ${ }^{1}$. En 1872, la Faculté de médecine de Berne comptait trois étudiantes; un an plus tard, elles étaient déjà 29 dont 27 Russes ${ }^{2}$.

Pour ce qui concerne Genève, la loi sur l'instruction publique d'octobre 1872 qui transformait l'ancienne Académie en Université stipulait que «les conditions d'entrée à l'Université sont les mêmes pour les deux sexes». Par voie de conséquence la Faculté de médecine, qui accueillit ses premiers étudiants en 1876, fut d'emblée mixte ${ }^{3}$. Et nombreuses seront les étudiantes, surtout slaves, souvent israélites, qui viendront s'y former: une première obtint le doctorat en 1883, une deuxième en 1889, alors que durant la décennie en question, 41 diplômes sont décernés à des hommes. Pendant la décennie allant de 1890 à 1899,26 doctorats en médecine sur un total de 141 
vont à des femmes, illustrant à la fois le développement d'une jeune faculté et la proportion d'abord modeste, puis croissante des femmes parmi les étudiants. Leurs études terminées, elles quittaient la Suisse, où peu de débouchés s'offraient à elles pour exercer leur métier. On ne note pas une seule Suissesse de naissance parmi les étudiantes qui reçurent avant 1900 le doctorat en médecine de l'Université de Genève.

\section{La porte fermée}

Les Radicaux, qui façonnèrent la politique genevoise au cours de la seconde moitié du XIX $\mathrm{XI}^{\mathrm{e}}$ siècle, avaient obtenu que l'université s'ouvrit largement aux étrangers. Il demeurait par contre difficile pour ceux et celles qui en sortaient diplômés d'exercer. Dans bien des milieux, on était en effet disposé à voir se former à Genève des universitaires pour la lointaine Russie, mais on répugnait à les voir s'installer durablement en ville et y concurrencer les médecins du cru. De ce point de vue, la vocation académique de Genève au siècle dernier en rappelle une autre alors en plein essor : l'hôtelière.

Henriette de Joudra (1856-1928) fut une exception. Originaire de Vitebsk, native de St-Petersbourg, elle s'inscrivit d'abord en Faculté des lettres et des sciences en 1875-76. L'année suivante, elle fit partie de la première volée d'étudiants de notre Faculté de médecine, d'abord comme auditrice puis, de 1877 à 1881, en qualité d'étudiante régulière. Elle soutint sa thèse de doctorat, Contribution à l'étude clinique du bruit de galop, le 27 juin 1883. Deux mois plus tard elle épousait un camarade d'étude, de quelques années son aîné, le docteur Charles Eugène Saloz, originaire de Moudon. Elle devint ainsi, par mariage, Suissesse. Mme de Joudra et son mari s'installèrent dans le même bâtiment du quartier de Rive, chacun ayant sa propre consultation. Elle avait surtout une clientèle féminine et une activité obstétricale, pédiatrique et gynécologique. Deux fils naîtront de leur mariage, Jacques en 1886 et Charles en 1888, qui deviendront à leur tour médecins après des études entreprises à l'Université de Genève. Une photo tardive du couple Saloz-de Joudra les montre fixant l'objectif d'un regard sérieux, voire sévère ${ }^{4}$.

Pratiquer la médecine à Genève au siècle dernier en étant femme et étrangère (même mariée à un Suisse) pouvait vous valoir la reconnaissance de vos patients mais ne vous ouvrait pas les portes des salons de la société genevoise. Henriette de Joudra en fit l'expérience lorsqu'elle tenta de devenir 


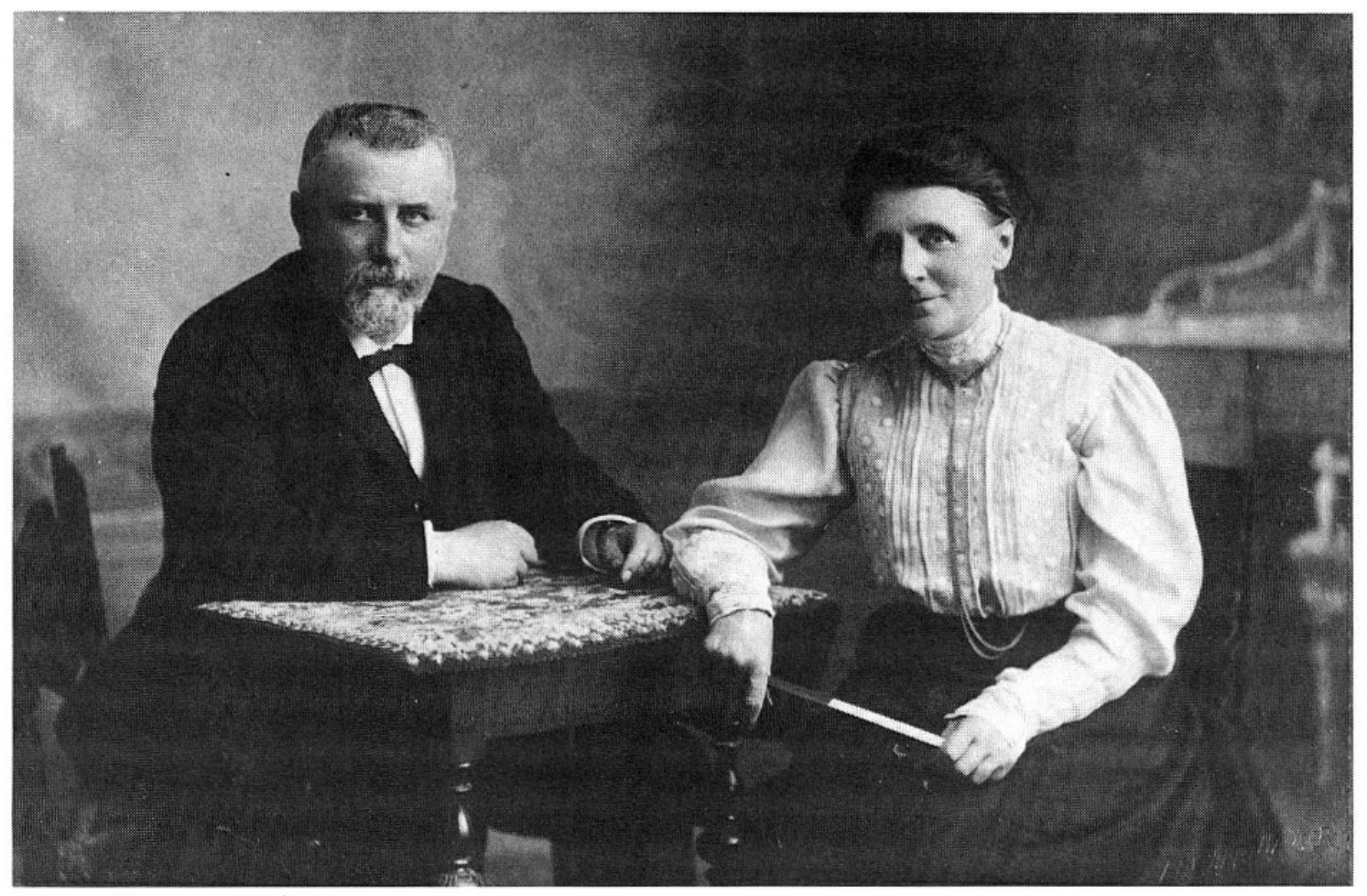

Les époux Charles Saloz et Henriette de Joudra.

membre de la Société médicale de Genève. Sa candidature créa quelques remous dont se fait trop pudiquement écho un rapport annuel: «Sur la proposition de quelques membres, notre bureau s'est réuni et nous avons même tenu une séance extraordinaire pour examiner l'opportunité de quelques modifications à nos règlements. Le résultat de ces réunions a été que, pour le moment, aucun changement n'est nécessaire» ${ }^{5}$. Ce texte cryptique s'éclaire au regard d'un autre - paru en 1923 - et qui rapporte le même épisode comme suit: «En octobre 1894, la question [des femmes médecins] fut posée à propos d'une candidature féminine qui avait été refusée précédemment, et donna lieu, dans une séance extraordinaire, à une vive discussion, qui se termina par la décision de l'ajournement indéfini» ${ }^{6}$. Charles Saloz, quant à lui, refusa d'entrer dans une Société qui avait fermé ses portes à son épouse.

Cette page de l'histoire de la médecine à Genève devait être tournée en janvier 1913, quand Marie Radmilovitch - une Russe qui avait aussi étudié la médecine à Genève et y avait obtenu le doctorat en 1905 - fut admise à la Société médicale. Elle avait épousé le docteur Bélisaire Huguenin de Berne et le couple résidait alors à Genève. En 1914, Huguenin fut rappelé à Berne 
pour y devenir professeur à la Faculté de médecine vétérinaire. C'est dire que sa femme ne fut membre de la Société Médicale que pendant une année. Son cas ne nous retiendra donc pas davantage. Il illustre l'évolution qui s'était faite en deux décennies.

\section{Deux destins}

Il fallut attendre le début de la décennie suivante pour voir à nouveau des femmes parmi les membres de la Société médicale. En 1921, deux personnalités de premier plan, Marguerite Champendal (1870-1928) et Lina Stern (1878-1968), furent accueillies d'un coup.

Née à Genève d'une famille suisse, influencée par l'exemple d'un père pasteur et d'un oncle médecin ${ }^{7}$, Marguerite Champendal choisit d'étudier la médecine malgré l'opposition initiale de sa famille. Elle s'inscrivit à la Faculté de médecine de Genève en 1892-93. Elle fit ses stages à la maternité, soutint en 1900 une thèse de doctorat - sur les varices congénitales - et consacra sa vie aux questions relatives à la santé de la mère et de l'enfant. Ses deux grandes réalisations sont La Goutte de lait et l'Ecole d'infirmières Le Bon Secours. La Goutte de lait, une transposition en 1901 d'une institution créée en 1894 au dispensaire de Belleville, à Paris, par le docteur Gaston Variot ${ }^{8}$ a perduré à Genève jusque bien après la seconde guerre mondiale. Elle fut bientôt complétée par une Pouponnière destinée aux bébés dont les mères étaient malades. Il fallait l'aide d'infirmières bénévoles; aussi Marguerite Champendal eut-elle l'idée de recruter des jeunes femmes dans les milieux aisés qui fussent disposées à œuvrer en tant que garde-malade, jetant ainsi les bases du Bon Secours. Le rôle qu'à joué Marguerite Champendal dans la création, puis dans la marche de cette école d'infirmières pendant plus de 20 ans, dépasse le cadre du présent travail ${ }^{9}$.

La vie de Lina Stern illustre un autre débouché possible pour les premières diplômées en médecine: la recherche scientifique et l'enseignement universitaire ${ }^{10}$. Née en Lettonie en 1878 dans une famille israélite, Lina Stern s'immatricula à la Faculté de médecine de Genève en 1897. Sa thèse de doctorat, présentée en 1903, avait pour sujet un travail expérimental. Entrée en tant qu'assistante en 1905 au Laboratoire de physiologie que dirigeait le professeur J.-L.Prévost (1838-1927), elle donna dès 1906 en qualité de privat-docent un enseignement portant sur les processus métaboliques. Ses recherches concernaient la biochimie et elle signa avec F. Batelli (1867-1941) 


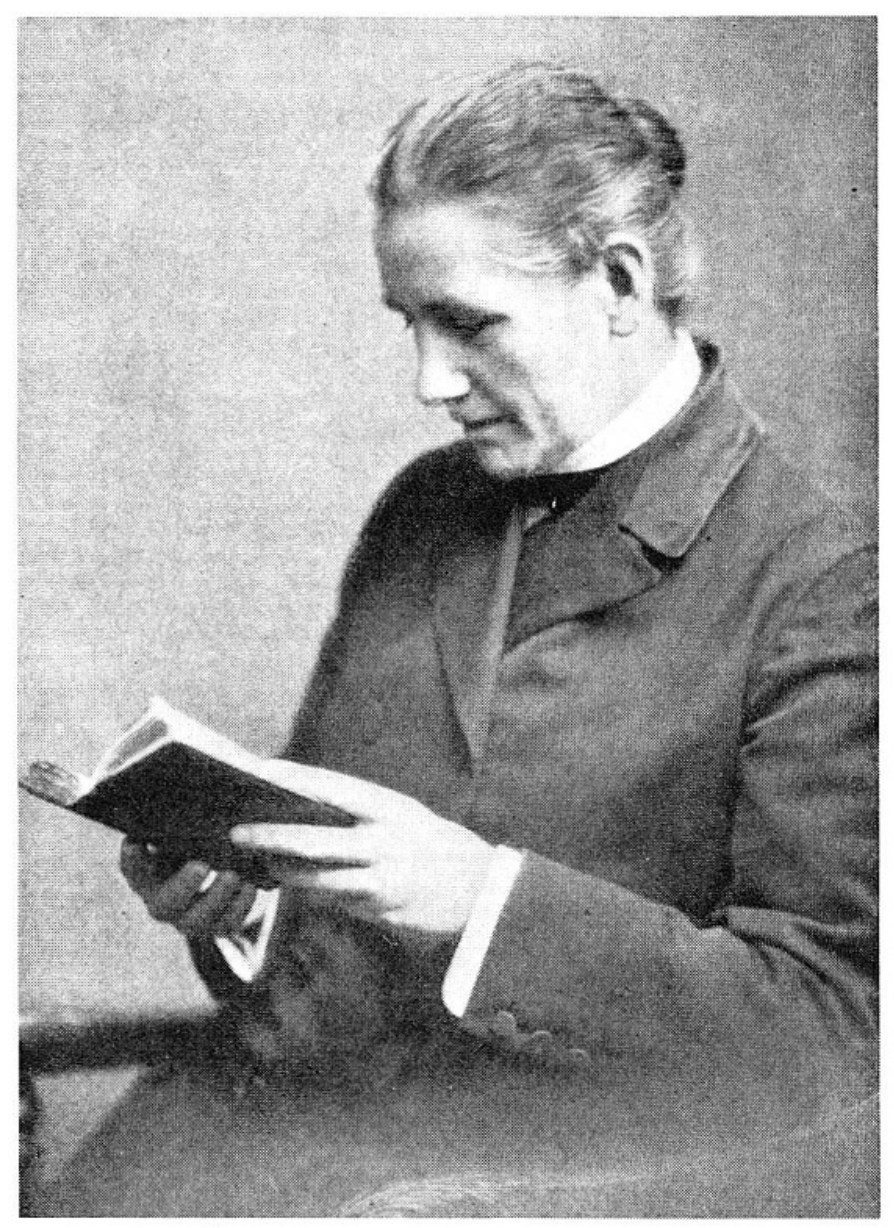

Marguerite Champendal (tiré de la publication mentionnée à la note 9).

- qui succéda en 1913 à Prévost à la chaire de physiologie genevoise - des travaux sur les enzymes et sur la respiration cellulaire.

La présence à l'Université de Genève de Lina Stern permit d'assurer dans de bonnes conditions, et conformément aux exigences d'un nouveau règlement des examens fédéraux de médecine, la séparation des enseignements de chimie physiologique et de physiologie. En février 1918, elle fut nommée "par vocation» professeur extraordinaire de chimie physiologique à la Faculté de médecine. Cet enseignement comprenait deux heures de cours par semaine durant le semestre d'hiver. Lina Stern cumulait cette fonction avec celle de chef des travaux à l'Institut de physiologie. Ce n'est qu'à partir de 1921 qu'elle reçut un modeste complément de salaire pour son enseignement de la chimie physiologique. Elle fut à ma connaissance la première femme à accéder au professorat dans une université suisse. ${ }^{11}$ 
Elle fut d'ailleurs longtemps la seule, et ce n'est qu'en 1947 que Kitty Ponse (1897-1982) - dont j'ai encore suivi l'enseignement et les travaux pratiques - fut nommée professeur extraordinaire d'endocrinologie à la Faculté des sciences. La première femme à porter le titre de professeur à l'Université de Zurich fut Hedwig Frey (1877-1938). Elle avait d'abord suivi l'Ecole normale puis, entrée à l'Université, elle s'était tournée vers l'anthropologie physique, domaine dans lequel elle obtint le doctorat en 1912. Elle entra alors comme assistante à l'Institut d'anatomie, où elle travailla durant toute sa vie professionnelle. Elle fut nommée professeur titulaire à la Faculté de médecine en 1924.

Gertrud Woker (1878-1968) fut la première femme professeur à l'Université de Berne. Fille d'un éminent professeur de théologie et d'histoire, elle étudia la chimie, la physique et la biologie, obtenant le doctorat en 1903 et le titre de privat-docent en 1907, après avoir effectué des stages postgradués chez van t'Hoff à Berlin et chez Sahli à Berne. A partir de 1911, elle dirigea le laboratoire de biologie physico-chimique qu'elle avait créé. Elle fut professeur extraordinaire de 1933 à 1951, année où elle prit sa retraite. Comme les premières recherches de Lina Stern, les travaux de recherche de Gertrud Woker concernent le métabolisme intermédiaire ${ }^{12}$.

Vers l'époque où elle fut nommée professeur à Genève, Lina Stern s'engagea dans un nouveau domaine de recherches. Des expériences faites avec de nombreuses substances injectées par voie sanguine lui avaient permis de constater que quelques-unes seulement passaient dans le tissu nerveux. Dans une conférence à la Société médicale de Genève, elle proposa l'hypothèse selon laquelle le cerveau serait protégé contre la pénétration de certaines substances par une sorte de filtre, auquel elle donna le nom de «barrière hémato-encéphalique» ${ }^{13}$. Ce concept connut une grande vogue et le terme qu'elle forgea est encore en usage jusqu'à ce jour.

Appelée à enseigner la physiologie à la Faculté de médecine de Moscou en 1925, Lina Stern vécut le reste de sa longue vie en Union Soviétique. Elle conserva pourtant de nombreux liens avec Genève et revenait parfois dans notre ville, du moins jusque vers 1934, année où elle fut élue membre de l'Académie des Sciences de l'URSS ${ }^{14}$. En 1938, elle entrait dans les rangs du parti communiste. Les dernières années du règne de Staline furent difficiles pour elle et, de 1949 à 1954, elle dut vivre en résidence surveillée. Après la mort du dictateur, elle fut réhabilitée et retrouva ses titres et privilèges d'académicienne. Elle décéda au printemps 1968. Huit ans plus tôt, elle avait reçu le doctorat honoris causa en sciences de l'Université de Genève ${ }^{15}$. 
Marguerite Champendal était morte en octobre 1928, âgée de 58 ans seulement. L'année précédente, Claire Debarge, une Suissesse qui avait obtenu le diplôme fédéral de médecine en 1923 à Genève, était devenue membre de la Société médicale; elle fut missionnaire en Afrique. Seules trois autres femmes furent admises dans la décennie suivante: Renée-Alice Girod en 1930, Jeanne Stephani-Cherbuliez en 1931 et Thérèse Chausse-Kling en $1934{ }^{16}$. Il n'est pas sans intérêt de noter que toutes trois sont Suissesses, que deux d'entre elles sont mariées et qu'elles sont admises 4 à 8 ans après avoir obtenu leur diplôme, tandis que Marguerite Champendal et Lina Stern ne le furent respectivement que 21 et 18 ans après leur graduation. Cette évolution traduit une timide et lente ouverture des membres de la Société médicale envers leurs consœurs; notons cependant que c'est une minorité qui y entre puisqu'une dizaine de femmes médecins exerçaient à Genève vers la fin des années vingt ${ }^{17}$.

Les conflits qui avaient parfois opposé l'Université à la corporation médicale sur la question de la féminisation de la profession devaient d'ailleurs progressivement s'atténuer ${ }^{18}$. Le fait que le nombre d'étudiantes slaves qui venaient entreprendre leurs études à l'Université de Genève déclina dès avant le déclenchement de la première guerre mondiale ${ }^{19}$, et s'effondra ensuite, tandis qu'augmentait peu à peu le nombre de Suissesses qui s'immatriculaient en Faculté de médecine, a certainement contribué à cette évolution. 
1 Rohner H., Die ersten 30 Jahre des medizinischen Frauenstudiums an der Universität Zürich 1867-1897, in Zürcher medizingeschichtliche Abhandlungen No 89, Juris Verlag, Zurich, 1972. Bonner T. N., Pioneering in women's medical education in the Swiss universities 1864-1914, Gesnerus 45, pp.461-474, 1988.

2 Bachmann B. \& Bradenahl E., Medizinstudium von Frauen in Bern 1871-1914, InauguralDissertation, Bern, 1990.

3 Dreifuss J. J., L'accès des femmes aux études médicales. Le premier demi-siècle (18761925) à Genève, in Cahiers de la Faculté de médecine, Université de Genève, No 14, mars 1987, pp. 13-27.

4. Madame R. Büchler, une petite fille d'Henriette de Joudra, m'a aimablement remis cette photographie.

5 Dr Cordès, Rapport sur la marche de la Société médicale de Genève en 1894, in Revue médicale de la Suisse romande, Quinzième année, 1895, p. 110.

6 Centenaire de la Société médicale de Genève 1823-1923. Notice historique par les Drs Picot et Thomas. Imprimerie Sonor, Genève, 1923, p. 72.

7 Marguerite Champendal a dédié sa thèse de doctorat à la mémoire de son père et de son grand'oncle, Charles-Elie Figuière (1814-1893), docteur en médecine de Paris, accoucheur, médecin adjoint du Bureau de salubrité, membre du Conseil administratif de la ville de Genève.

8 Gaston Variot (1855-1930), médecin et patriote français, un des pères de la puériculture. Il s'appuya sur le pastorisme pour diminuer la mortalité infantile et pour enrayer le recul démographique de la France en créant notamment, avec des fonds privés, le dispensaire de Belleville et la Goutte de Lait.

9 Brocher Sara, La doctoresse Champendal. Association du Bon Secours, Genève, 1968. Cette hagiographie, d'abord publiée en 1951, comporte quelques textes et lettres de Mlle Champendal.

10 Posternak Jean, La physiologie à la Faculté de Médecine à Genève, in Centenaire de la Faculté de Médecine de l'Université de Genève (1876-1976). Documents rassemblés par M. Cramer et J.Starobinski. Bio-bibliographies par M.-A. Barblan, Editions Médecine et Hygiène, Genève, 1978, pp. 54-56.

11 Marguerite Champendal fut privat-docent à la Faculté de Médecine à partir de 1912.

12 Je remercie le Dr Heinz Balmer, privat-docent et collaborateur permanent de l'Institut d'histoire de la médecine de l'Université de Zurich, d'avoir attiré mon attention sur les carrières académiques de Mlles H. Frey et G. Woker.

13 Le texte de cette conférence faite le 21 avril 1921 fut publié dans Schweizer Archiv für Neurologie und Psychiatrie, Le liquide céphalo-rachidien au point de vue de ses rapports avec la circulation sanguine et avec les éléments nerveux de l'axe cérébrospinal, volume 8, 1921, pp. 215-232. Trois articles suivirent, cosignés par R. Gautier, sous le titre général de Recherches sur le liquide céphalorachidien, in Archives internationales de Physiologie, vol. 17, pp. 139-192 et 391-448; vol. 18, pp.403-436, 1921-1923.

14 De 1927 à 1933, elle présentait parfois à la Société de Biologie de Paris des travaux qu'elle et ses collaborateurs avaient réalisés à Moscou. De nombreuses notes, rédigées en français figurent dans les Compte-rendus hebdomadaires de cette Société. Elle assista au quator- 
zième Congrès international de physiologie qui se tint à Rome en 1932 et elle séjourna à cette occasion à Genève, où elle rendit visite à Edouard Claparède, qui l'avait précédée de peu d'années sur les bancs de l'Ecole de médecine genevoise.

15 La collation eut lieu en l'absence de l'intéressée, alors octogénaire, lors du Dies Academicus, le 2 juin 1960. Une allocution fut prononcée par le doyen Fernand Chodat qui souligna l'importance des recherches de Lina Stern sur les enzymes oxydatifs et sur la «barrière hémato-encéphalique».

16 Je dois ces renseignements au Dr Roger Mayer, archiviste de la Société médicale de Genève, que je tiens à remercier.

17 Gourfein-Welt L., in Das Frauenstudium an den Schweizer Hochschulen. Schweizerischer Verband der Akademikerinnen, Zurich, 1928, chapitre «Genève», pp. 135-168. On trouvera dans ce texte de nombreuses données sur les premières femmes y ayant exercé une profession médicale, en particulier pp. 145-151.

18 La lutte pour l'amélioration des conditions matérielles et professionnelles de la pratique médicale à Genève fut en fait surtout l'affaire de l'Association des médecins du canton de Genève, créée en 1892. La Société médicale n'était pas un groupe de pression, mais davantage un lieu de rencontres et de formation continue. Voir Ehrenström Ph., Association médicale: à l'aube d'un centenaire, Revue médicale de la Suisse romande 111, 1991, pp. 81-83.

19 Mysyrowicz L., Les étudiants «orientaux» en médecine à Genève (1876-1914), Gesnerus 34, 1977, pp. 207-212. 


\section{Summary}

The professional career of some of the first women physicians graduated from the University of Geneva Medical School

Amongst the first students enrolled at the Faculty of Medicine of Geneva in the last decades of the 19th century were many women, mostly originating from Eastern Europe. After graduation, only few remained in Geneva. The life of three early graduates illustrates the difficulties encountered by women doctors at the turn of the century in Geneva and some of the ways to overcome the resistance encountered. Henriette de Joudra (1856-1928) married a Swiss physician and practised in town; the Société médicale de Genève however never granted her membership. Marguerite Champendal (1870-1928), one of the first Swiss nationals to graduate, created a school for nurses that she directed until her untimely death. Lina Stern (1878-1968), of Russian origin, turned after graduation to laboratory research and to university teaching: she performed original research in biochemistry and in the neurosciences. From 1918 onwards she was the first woman having professional rank at the University of Geneva. After 1925 she pursued a brilliant academic career in the Soviet Union.

\section{Zusammenfassung}

\section{Die ersten Studentinnen der Medizinischen Fakultät und ihre Berufstätigkeit in Genf}

Seit ihrer Eröffnung (1876) gewährte die Medizinische Fakultät der Universität Genf den Frauen Zutritt. Von den zahlreichen Studentinnen, vor allem aus den slawischen Ländern, blieben aber nur wenige nach dem Abschluss ihres Studiums in Genf, wo ihnen die Ärzteschaft eher feindlich gegenüberstand. Drei Beispiele beleuchten die Schwierigkeiten der ersten Ärztinnen in Genf. Henriette de Joudra (1856-1928) heiratete einen Schweizer Arzt und widmete sich gleichzeitig ihrer Familie wie der ärztlichen Praxis; die Ärztegesellschaft verweigerte ihr jedoch die Mitgliedschaft. Marguerite Champendal (1870-1928), eine der ersten Medizinerinnen schweizerischer Herkunft, sorgte für junge Mütter und Säuglinge und gründete die Krankenpflegeschule «Le Bon Secours». Lina Stern (1878-1968) wurde 1918 zur ausserordentlichen Professorin für physiologische Chemie ernannt und ist damit die erste Medizinprofessorin an einer schweizerischen Universität. 1925 folgte sie einem Ruf nach Moskau.

Professeur J. J. Dreifuss

Département de Physiologie

Centre Médical Universitaire

1, Rue Michel Servet

CH-1211 Genève 4 